\title{
Review of "Open Science"
}

\author{
Paola Masuzzo \\ 1 Ins titute for Globally Distributed Open Research and Education (IGDORE)
}

This is a nice definition. I love the 'long-tem preservation' of it.

However, I have the feeling that 'data' and 'results' are not enough. We could argue

forever as to what is data and what is not, and perhaps this is why a unique, agreed-upon definition of Open Science does not exist yet. Is software code data? If yes, this definition is exhaustive. If not, I would explicitly add software code.

And what about protocols? Tools? Physical samples, whenever possible?

Methodologies?

I like the definition, it's sweet and short, but perhaps a bit too short :) 